\title{
Performance Enhancement of Photoconductive Antenna Using Saw-Toothed Plasmonic Contact Electrodes
}

\author{
Xingyun Zhang ${ }^{1}$, Fangyuan Zhan ${ }^{2}$, Xianlong Wei ${ }^{2}$, Wenlong $\mathrm{He}^{3}$ and Cunjun Ruan $1,4, *$ (D) \\ 1 School of Electronic and Information Engineering, Beihang University, Beijing 100191, China; \\ luckyzhang@buaa.edu.cn \\ 2 Department of Electronics, Peking University, Beijing 100871, China; 1901111193@pku.edu.cn (F.Z.); \\ weixl@pku.edu.cn (X.W.) \\ 3 College of Electronics and Information Engineering, Shenzhen University, Shenzhen 518061, China; \\ wenlong.he@szu.edu.cn \\ 4 Beijing Key Laboratory for Microwave Sensing and Security Applications, Beihang University, \\ Beijing 100191, China \\ * Correspondence: ruancunjun@buaa.edu.cn
}

check for updates

Citation: Zhang, X.; Zhan, F.; Wei, X.; He, W.; Ruan, C. Performance Enhancement of Photoconductive Antenna Using Saw-Toothed Plasmonic Contact Electrodes. Electronics 2021, 10, 2693. https:/ / doi.org/10.3390/electronics10212693

Academic Editors: George Kyriacou, George Trichopoulos and

Filippos Farmakis

Received: 13 October 2021

Accepted: 1 November 2021

Published: 4 November 2021

Publisher's Note: MDPI stays neutral with regard to jurisdictional claims in published maps and institutional affiliations.

Copyright: (c) 2021 by the authors. Licensee MDPI, Basel, Switzerland. This article is an open access article distributed under the terms and conditions of the Creative Commons Attribution (CC BY) license (https:/ / creativecommons.org/licenses/by/ $4.0 /)$.

\begin{abstract}
A photoconductive logarithmic spiral antenna with saw-toothed plasmonic contact electrodes is proposed to provide a higher terahertz radiation compared with the conventional photoconductive antenna (PCA). The use of saw-toothed plasmonic contact electrodes creates a strong electric field between the anode and cathode, which generates a larger photocurrent and thereby effectively increases the terahertz radiation. The proposed PCA was fabricated and measured in response to an $80 \mathrm{fs}$ optical pump from a fiber-based femtosecond laser with a wavelength of $780 \mathrm{~nm}$. When the proposed antenna is loaded with an optical pump power of $20 \mathrm{~mW}$ and a bias voltage of $40 \mathrm{~V}$, a broadband pulsed terahertz radiation in the frequency range of $0.1-2 \mathrm{THz}$ was observed. Compared to the conventional PCA, the THz power measured by terahertz time domain spectroscopy (THz-TDS) increased by an average of 10.45 times.
\end{abstract}

Keywords: photoconductive antenna (PCA); saw-toothed; plasmonic contact electrodes; terahertz radiation

\section{Introduction}

The terahertz $(\mathrm{THz})$ band lies in the frequency range between the millimeter wave and far infrared, describing the electromagnetic spectrum ranges from $100 \mathrm{GHz}(3 \mathrm{~mm})$ to 10 $\mathrm{THz}(30 \mu \mathrm{m})[1,2]$. Terahertz technology has attracted much attention due to its widespread applications in non-destructive testing, spectroscopy, material characterization, medical imaging, security imaging, and ultrafast data transfer [3-10]. Therefore, it is imperative to develop high-power terahertz sources. Among various techniques for terahertz generation, photoconductive antennas (PCAs) have been extensively utilized to generate pulsed terahertz radiation [11-13]. The main reason is the development of ultrashort pulse lasers and low-temperature grown gallium arsenide (LT-GaAs). LT-GaAs can provide broad bandwidth due to its short carrier recombination time (less than 1 ps). In addition, the quantum efficiency of the photoconductive terahertz emitters is not limited by the Manley Rowe limit [14], and the quantum efficiency is higher than that of the terahertz radiation sources based on the optical rectification method. Therefore, technically speaking, the conversion efficiency of photoconductive antennas from optics to terahertz can reach $100 \%$. However, the poor quantum efficiency of conventional photoconductive terahertz emitters limits the terahertz output power.

Many techniques have been developed to address this limitation [15-28]. Large-area photoconductive emitters based on plasmonic contact electrodes have been proposed to achieve high terahertz power levels [17]. Combining the plasmonic contact electrodes with the device area provides a much stronger time-varying dipole moment in response to an 
optical pump, which efficiently enhances the optical-to-terahertz conversion efficiency. By using the strongly coupled piezoelectric behavior in a GaN layer, a metal-semiconductormetal photoconductive terahertz emitter with enhanced output $\mathrm{THz}$ power was proposed in [18]. LT-GaAs thin-film-based PCAs were fabricated and validated to offer a high peak $\mathrm{THz}$ radiation signal compared with conventional PCAs [19]. The proposed LT-GaAs thin-film configuration was used to increase the number of photo-generated carriers, which contributed to a high optical-to-terahertz conversion efficiency. A log-periodic $\mathrm{THz}$ photoconductive antenna coupled with a silver nanoantenna array was put forward in [20]. By changing the size of silver nanoantennas and their arrangement, an increase in opticalto-terahertz conversion efficiency was achieved. An all-dielectric nanograting-assisted PCA with enhanced THz radiation power was proposed and demonstrated in [21]. The integrated nanograting can increase the number of photocarriers and the bias electric field loaded on the photocarriers, achieving a nearly uniform enhancement of terahertz power. The plasmonic contact electrodes were also introduced to generate high terahertz radiation power from a photoconductive terahertz emitter based on a bow-tie antenna [27] and logarithmic spiral antenna [28]. Despite the fact that these previous research prototypes have been demonstrated to enhance the $\mathrm{THz}$ generation from PCAs, the saw-toothed plasmonic contact electrodes we designed have not yet been studied.

In this paper, a broadband photoconductive logarithmic spiral antenna with sawtoothed plasmonic contact electrodes for $\mathrm{THz}$ generation enhancement is proposed. The logarithmic spiral antenna is mainly used to achieve a broadband output [29-31]. By using the saw-toothed plasmonic contact electrodes, a strong electric field can be formed between the anode and cathode of the proposed antenna, contributing to a larger terahertz radiation generated from the proposed PCA. We have fabricated prototypes of the developed PCA and a conventional PCA with the same size and structure, and characterized the former so as to demonstrate its ability to offer high power terahertz radiation through experiments. The experimental results verify the broadband and enhanced $\mathrm{THz}$ radiation characteristics of the proposed antenna.

\section{Materials and Methods}

A photoconductive terahertz emitter consists of an external optical pump, an antenna located on a photoconductor, and a bias voltage. When a sub-picosecond optical pump is incident on an ultrafast photoconductive material, electron-hole pairs are generated, and these electron-hole pairs move under the applied bias electric field to generate a photocurrent. The induced photocurrent follows the envelope of the optical pump, driving the antenna to generate terahertz radiation.

Figure 1a shows a microscope image of the proposed photoconductive antenna. The proposed antenna was fabricated on a $350 \mu \mathrm{m}$ thick semi-insulating GaAs (SI-GaAs) substrate with a relative permittivity of 12.9 , and the end-to-end distance between the anode and cathode was $4 \mu \mathrm{m}$. The width of the bias lines was set at $6 \mu \mathrm{m}$, which had almost no effect on the radiation characteristics of the proposed PCA. Compared with LT-GaAs, the SI-GaAs we used in the paper is more easily accessible and less expensive. The logarithmic spiral antenna was used to provide a broadband radiation characteristic and high radiation efficiency. The logarithmic spiral antenna consists of two winding conductors, and the edges can be calculated by the equations [29]:

$$
r_{1}=r_{0} e^{a \phi}, r_{2}=r_{0} e^{a(\phi-\delta)}, r_{3}=r_{0} e^{a(\phi-\pi)}, r_{4}=r_{0} e^{a(\phi-\delta-\pi)},
$$

where angle $\delta$ is the arm width, $\phi$ is the azimuth angle, and $r_{0}$ and a are constants that control the size of the terminal area and the growth rate of the spiral, respectively. Here we set $\delta=1.5$ and $a=0.31$. The operating frequency of a logarithmic spiral antenna is 
determined by the radius of the inner and outer rings and the growth rate of spiral. The low-frequency limit is calculated so:

$$
\begin{gathered}
\lambda_{\text {low }}=2 r_{0}\left[e^{a \phi(\max )}-e^{a(\phi(\max )-\pi)}\right] e^{-a \frac{4}{\pi}} \sqrt{\frac{1}{a^{2}}+1}, \\
f_{\text {low }}=\frac{c_{0}}{\lambda_{\text {low }}} \frac{1}{\sqrt{\left(\varepsilon_{r}+1\right) / 2}},
\end{gathered}
$$

where $\phi(\max )$ represents the angle of the end of spiral, $c_{0}$ is the speed of light, and $\varepsilon_{r}=12.9$ is the relative permittivity of GaAs. The high-frequency limit is calculated so:

$$
\begin{gathered}
\lambda_{\text {high }}=2 r_{0}\left[e^{a(\phi(0)+\pi)}-e^{a \phi(0)}\right] e^{a \frac{4}{\pi}} \sqrt{\frac{1}{a^{2}}+1}, \\
f_{\text {high }}=\frac{c_{0}}{\lambda_{\text {high }}} \frac{1}{\sqrt{\left(\varepsilon_{r}+1\right) / 2}},
\end{gathered}
$$

where $\phi(0)$ represents the start of the spiral. By using Equations (2)-(5), the parameters $r_{0}=$ $5 \mu \mathrm{m}$ and $\phi(\max )=13$ can be calculated, enabling the proposed antenna to operate at 0.1 to $2 \mathrm{THz}$.

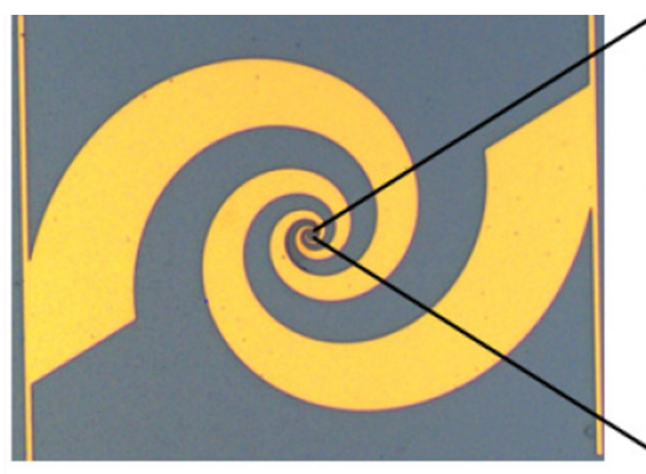

(a)

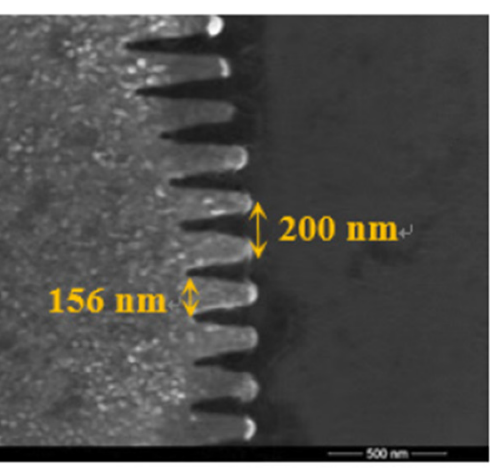

(b)

Figure 1. PCA with saw-toothed plasmonic contact electrodes and a conventional PCA. (a) Microscope image of the proposed PCA; (b) Scanning electron microscope of the designed saw-toothed plasmonic contact electrodes.

The scanning electron microscope (SEM) image of the saw-toothed plasmonic contact electrodes with a $200 \mathrm{~nm}$ pitch, $156 \mathrm{~nm}$ spacing, and $50 \mathrm{~nm}$ height is shown in Figure 1b. "Plasmonic" refers to the group of electron oscillations that occur in sub-wavelength sized metallic nanostructures when they are excited by an optical pump [32]. These oscillations can significantly increase the optical near field, allowing a large number of photo-carriers to arrive at the photoconductor contact electrodes during the terahertz oscillation period, thereby greatly contributing to the terahertz radiation. Compared with the plasmonic contact electrodes presented before, the designed saw-toothed plasmonic contact electrodes could form a stronger electric field due to the tip effect. The tip effect refers to the phenomenon in which, on the same charged conductor, compared with the smooth part, the surface charge density of the tip is larger, and the formed electric field is stronger. This occurs due to the fact that the induced photocurrent is proportional to the electric field, leading to a larger terahertz radiation generated by the proposed PCA. Figure 2 shows the microscope image of the traditional PCA, which was used as the reference to validate the radiation enhancement of our proposed antenna. 


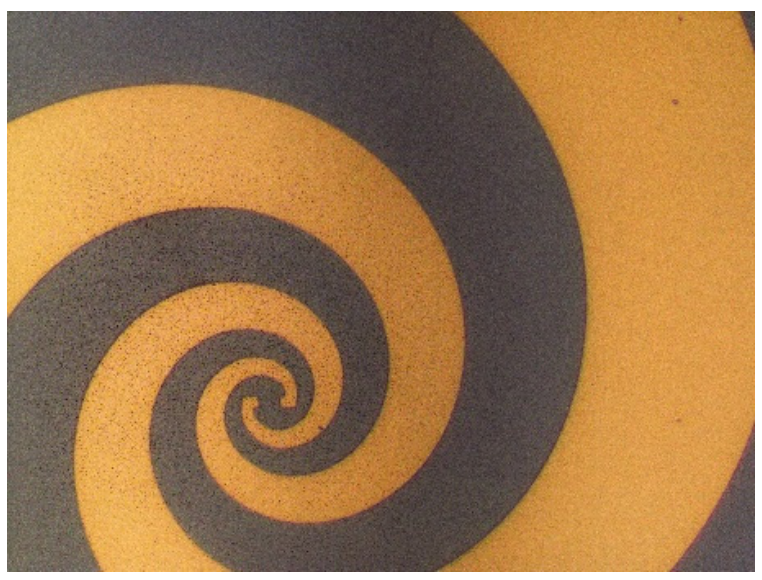

Figure 2. Microscope image of the conventional PCA.

\section{Experiments and Discussions}

We fabricated prototypes of the proposed PCA with saw-toothed plasmonic contact electrodes and a conventional photoconductive antenna on a SI-GaAs substrate. For the conventional PCA, the bias lines and antennas were patterned using electron beam lithography followed by a Ti/Au $(10 / 190 \mathrm{~nm})$ deposition and liftoff. For the PCA with designed saw-toothed plasmonic contact electrodes, the fabrication process began with the patterning of logarithmic spiral antennas and bias lines using electron beam lithography, followed by a $5 / 200 \mathrm{~nm} \mathrm{Ti} / \mathrm{Au}$ deposition and liftoff. The nanoscale saw-toothed plasmonic contact electrodes were patterned using electron beam lithography, followed by a deposition of $5 / 45 \mathrm{~nm} \mathrm{Ti} / \mathrm{Au}$ and liftoff.

Figure 3 shows the prototype of our proposed PCA. The fabricated device was then put on a hyper-hemispherical silicon lens, which collected and collimated the generated terahertz radiation from the rear side of the photoconductor. In order to apply the bias voltage to the fabricated PCA, an external PCB power supply board was required as shown in Figure 4. Gold wires were soldered onto the anode and cathode of the antenna and connected to the PCB power supply board. Then, two metal wires connected to the external bias module were soldered onto the other end of the PCB board.

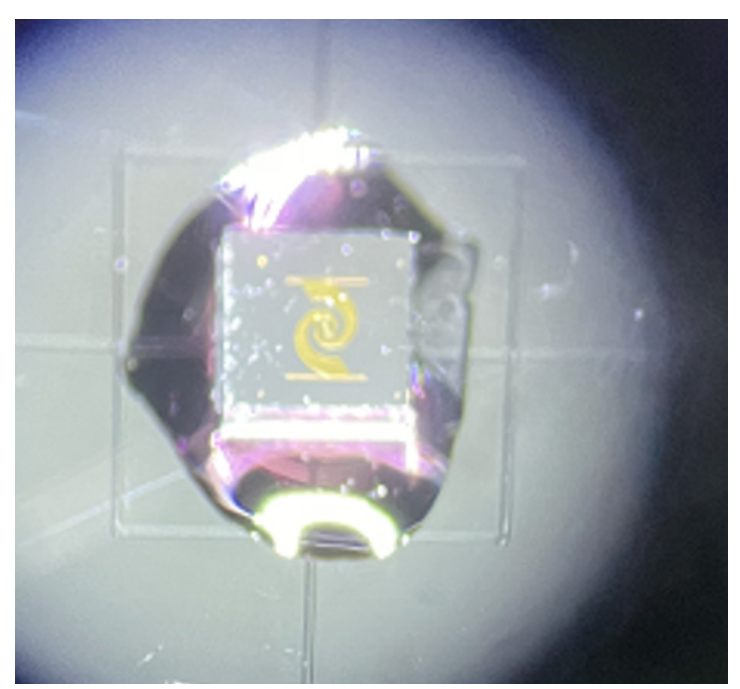

Figure 3. Photograph of the fabricated antenna under the microscope. 


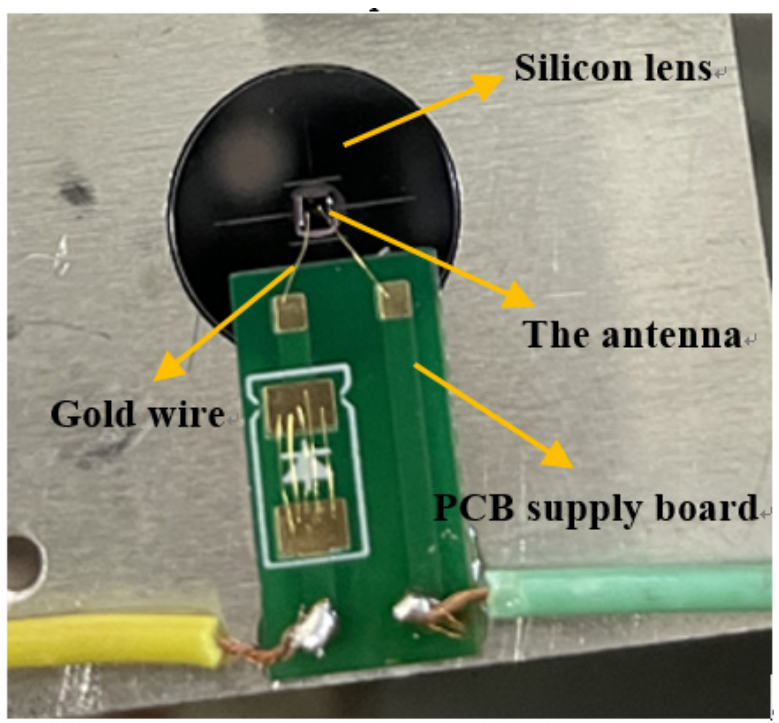

Figure 4. Photograph of the THz photoconductive emitter for measurement.

The THz time domain spectroscopy (TDS) system shown in Figure 5 was used to measure the proposed PCA and conventional PCA. The fabricated PCAs were placed at the focal point of the laser at the transmitter. Since the $\mathrm{THz}$ radiation signal generated by the antenna was very small, an ultra-low-noise current amplifier was used here to linearly amplify it.

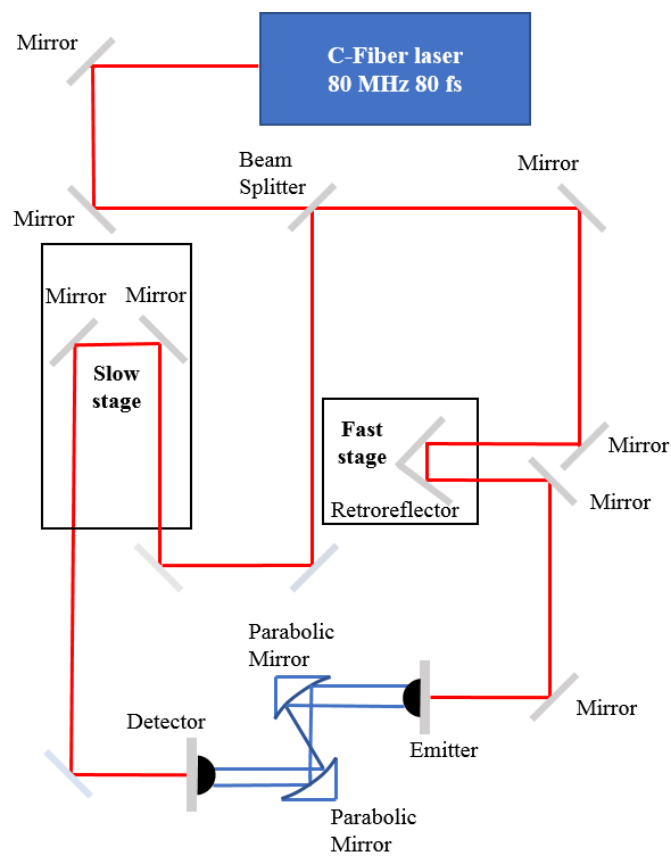

(a)

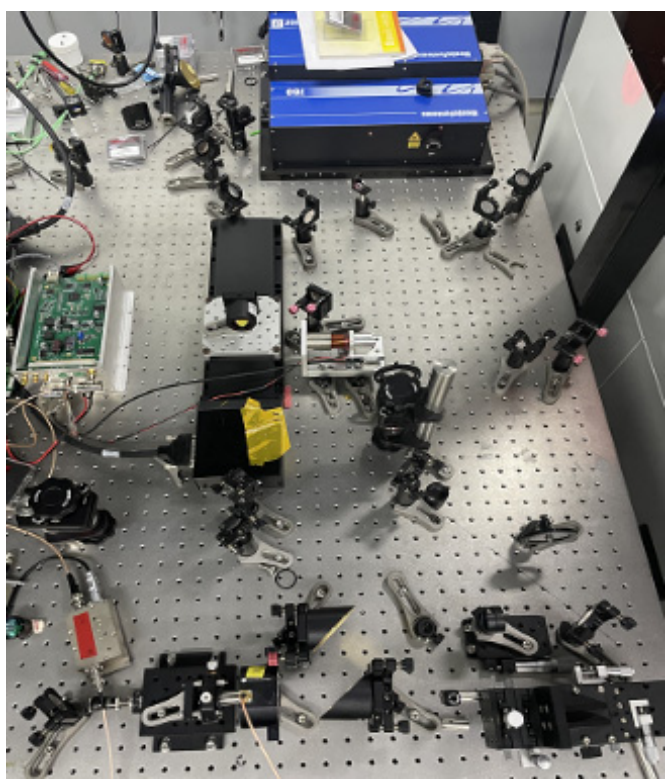

(b)

Figure 5. Measurement setup. (a) Schematic diagram; (b) photograph of the THz-TDS.

To characterize the proposed PCA with saw-toothed plasmonic contact electrodes and conventional PCA, radiation signals were measured in response to an incident optical pump from a fiber-based femtosecond laser at a wavelength of $780 \mathrm{~nm}$ with a repetition rate of $80 \mathrm{MHz}$ and a pulse width of $80 \mathrm{fs}$. The position of the circular pump spot was tuned to maximize the terahertz radiation. The measured $\mathrm{THz}$ radiation signals of the two 
prototype devices with a bias voltage of $40 \mathrm{~V}$ and an optical pump with a power of $20 \mathrm{~mW}$ are shown in Figure 6a. It can be observed that when the same optical pump power and bias voltage was applied, the measured peak-to-peak $\mathrm{THz}$ radiation signal of our proposed antenna with saw-toothed plasmonic electrodes showed 1.6 times growth compared to the reference PCA. The radiation power for pulsed emitters can be calculated as [18]:

$$
P_{\text {rad }}(\mathrm{t})=\frac{1}{2} I_{p h}^{2}(\mathrm{t}) R_{\text {rad }}(\omega),
$$

where $I_{p h}(\mathrm{t})$ is the photocurrent and $R_{r a d}(\omega)$ is the radiation resistance of the antenna. It should be noted that $I_{p h}(\mathrm{t})$ and $R_{r a d}(\omega)$ are frequency-dependent quantities in the pulse mode. Compared with the conventional PCA, we found that the proposed PCA provided a higher photocurrent level, indicating an obvious enhancement in radiation power due to the quadratic relation between photocurrent and radiation power.

Figure $6 \mathrm{~b}$ illustrates the $\mathrm{THz}$ power spectrums of both antennas. Unlike microwave and millimeter antennas whose frequency bandwidth depends on the $-10 \mathrm{~dB}$ range of S-parameters, the terahertz bandwidth of a PCA is usually described as the range of frequencies within which the measured signal strength in the frequency domain exceeds the noise level of the system. The measured radiation bandwidths of the two antennas were almost the same, between $0.1-2 \mathrm{THz}$, which is basically consistent with the theoretical calculation results. The obtained dynamic ranges of the proposed antenna and the reference antenna were $66 \mathrm{~dB}$ and $55 \mathrm{~dB}$, respectively.

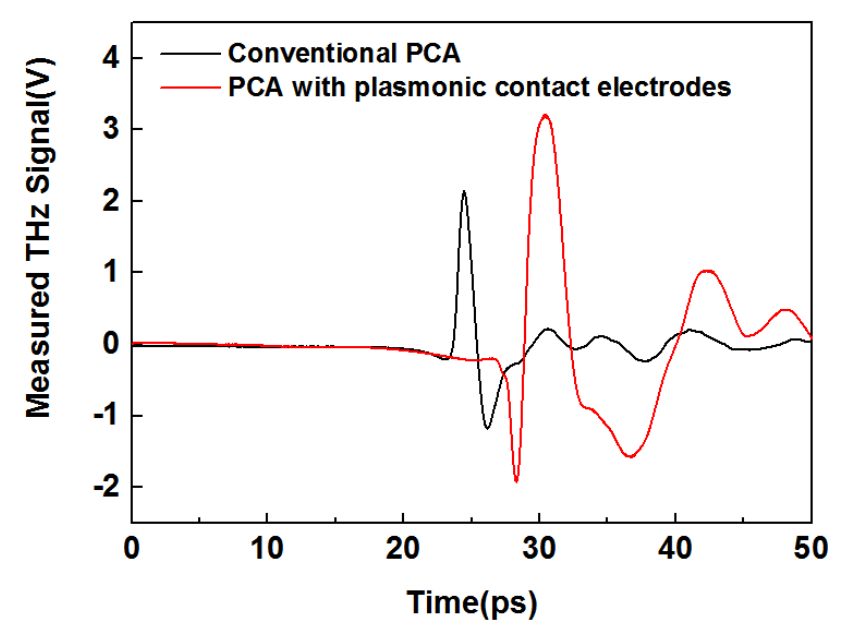

(a)

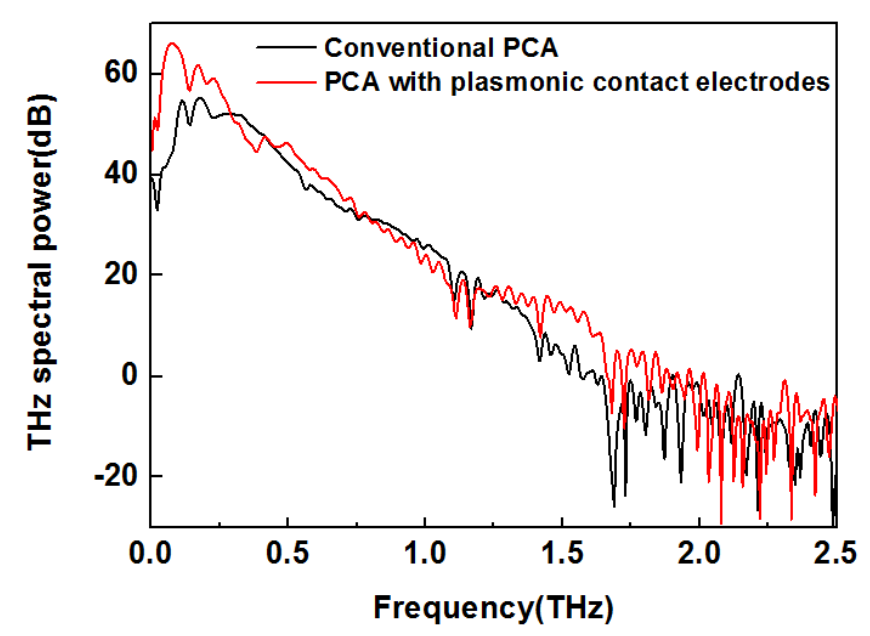

(b)

Figure 6. (a) The measured THz signals in the time domain and (b) THz power spectrums in the frequency domain for the proposed PCA with saw-toothed plasmonic contact electrodes and the conventional PCA.

Figure 7 shows the $\mathrm{THz}$ power improvement of the proposed antenna compared with the traditional PCA at different frequencies. As can be seen, a maximal power enhancement factor of up to 191 was realized at $0.06 \mathrm{THz}$ over the 0.1 to $1.6 \mathrm{THz}$ range. The THz power enhancement was about 10.45 on average over the 0.1 to $2 \mathrm{THz}$ range. The uneven enhancement can be related to the variation in the PCA impedance induced by the saw-toothed plasmonic contact electrodes [20]. It is worth noting that when we used the LT-GaAs as the substrate, a broader bandwidth and higher radiation power could be realized. 


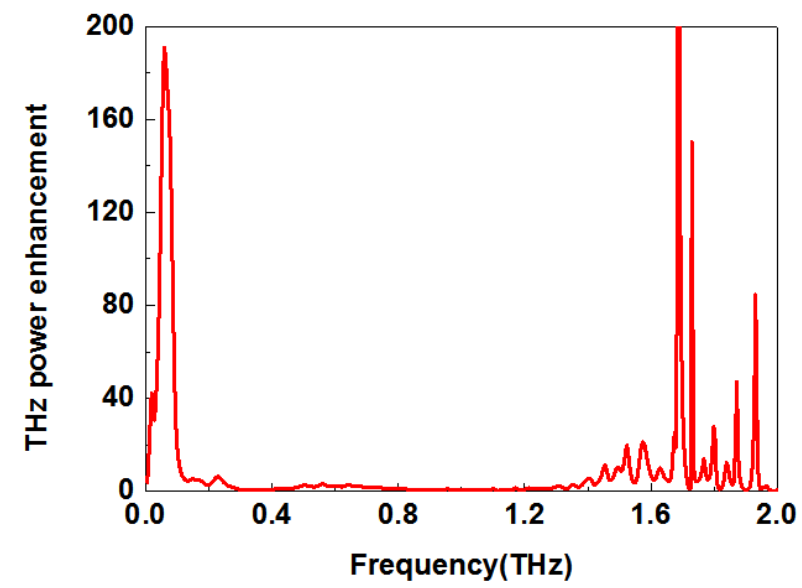

Figure 7. THz power enhancement of the proposed PCA compared to the conventional PCA at different frequencies.

Figure $8 \mathrm{a}$ shows the measured $\mathrm{THz}$ radiation signals as a function of optical pump powers. As the optical pump power increased from $5 \mathrm{~mW}$ to $30 \mathrm{~mW}$, the extracted peak to peak THz signal increased from $2.36 \mathrm{~V}$ to $6.08 \mathrm{~V}$, as shown in Figure $8 \mathrm{~b}$. We can see that our proposed PCA produces a higher $\mathrm{THz}$ radiation signal at the same optical pump power compared to the conventional PCA. At lower optical pump power levels, the measured $\mathrm{THz}$ radiation signal was significantly increased. This was because a larger number of photocarriers were generated near the saw-toothed plasmonic contact electrodes, which thereby exhibited higher photocurrent levels with the increasing optical pump powers. However, under high optical pump powers $(20-30 \mathrm{~mW})$, the enhancement effect was slightly degraded. This was due to the presence of the carrier-screening effect at a high optical pump power [33]. When a large number of electron-hole pairs are separated from each other, a radiation field opposite to the bias electric field was formed, which reduced the bias electric field, thus weakening the growth of the measured $\mathrm{THz}$ radiation signal.

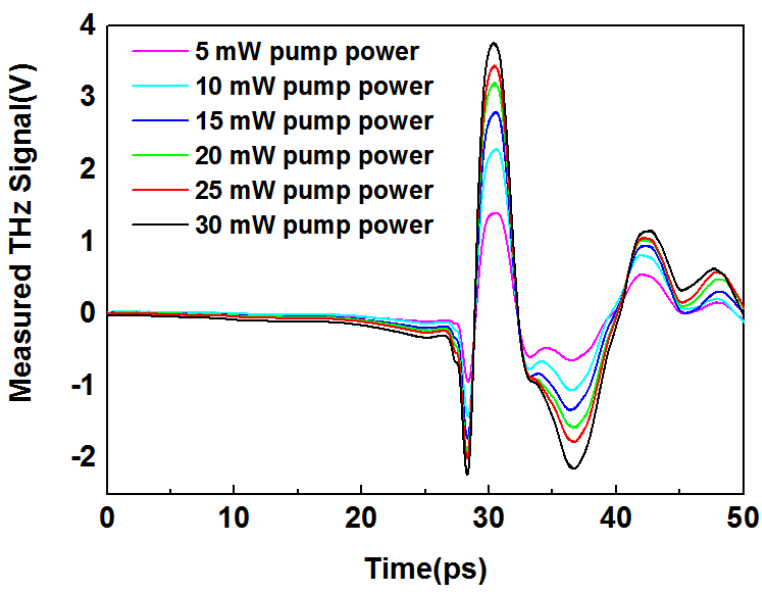

(a)

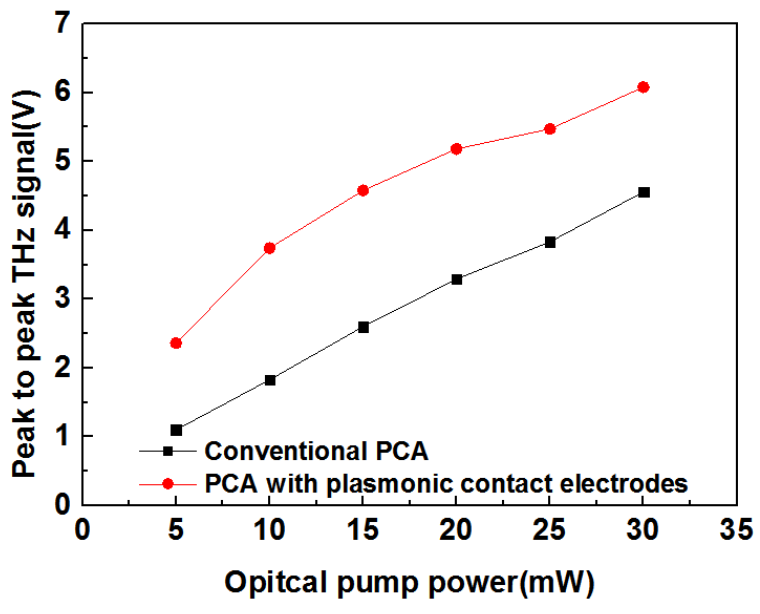

(b)

Figure 8. (a) The measured THz signals and (b) extracted peak-to-peak THz signals of the proposed PCA as a function of the optical pump power.

Figure 9 shows the measured $\mathrm{THz}$ power spectrums of the proposed PCA as a function of the optical pump power and bias voltage. As expected, the measured $\mathrm{THz}$ power amplitudes increased as the optical pump power and bias voltage increased. In addition, we also found that the radiation bandwidth of the proposed PCA was not affected by the 
optical pump power and bias voltage. The main reason was that the radiated spectrum was mainly determined by the spectral characteristics of the proposed PCA.

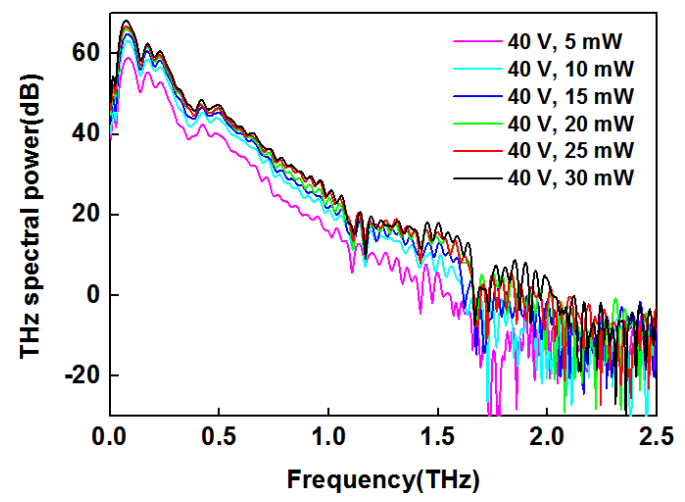

(a)

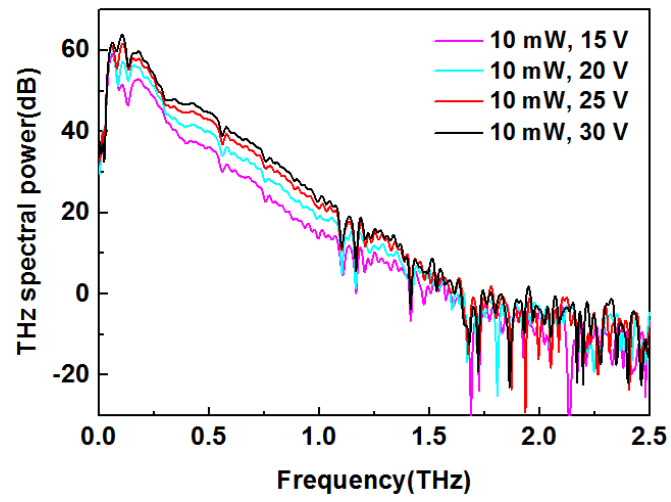

(b)

Figure 9. The measured $\mathrm{THz}$ power spectrums of the proposed PCA as a function of the (a) optical pump power and (b) bias voltage.

\section{Conclusions}

In conclusion, in this paper we proposed and validated the power enhancement of a photoconductive terahertz emitter based on a logarithmic spiral antenna with saw-toothed plasmonic contact electrodes. Due to the strong electric field generated by the designed saw-toothed plasmonic contact electrodes, a large number of photocarriers drifted to the photoconductor contact electrodes within a terahertz oscillation cycle to efficiently contribute to terahertz radiation. At an optical pump power of $20 \mathrm{~mW}$ and bias voltage of $40 \mathrm{~V}$, we generated $5.18 \mathrm{~V}$ of peak-to-peak THz radiation signal over $0.1-2 \mathrm{THz}$, which was 1.6 times that of the reference PCA. Compared with the reference PCA, our proposed PCA showed a $\mathrm{THz}$ power enhancement of 10.45 times on average over the $0.1-2 \mathrm{THz}$ range. The improved terahertz output power of the developed PCA makes it a good candidate for future time domain terahertz spectroscopy systems. Due to the limitation of the test platform, the radiated power of the proposed PCA has not been obtained. Our future work will aim to improve the THz-TDS and measure the radiated power of the proposed antenna.

Author Contributions: Conceptualization, X.Z. and C.R.; software, X.Z.; device fabrication, F.Z., X.Z. and X.W.; validation, X.Z., W.H. and C.R.; formal analysis, X.Z.; investigation, X.Z.; data curation, X.Z.; writing—original draft preparation, X.Z.; writing—review and editing, X.Z., C.R., F.Z. and X.W.; visualization, X.Z.; supervision, C.R.; project administration, C.R.; funding acquisition, C.R. and W.H. All authors have read and agreed to the published version of the manuscript.

Funding: This research was funded by the Natural Science Foundation of China, grant number 61831001, and by the Shenzhen Science and Technology Program, grant number KQTD20200820113046084.

Data Availability Statement: The data used to support the findings of this study are available from the corresponding author upon request.

Conflicts of Interest: The authors declare no conflict of interest.

\section{References}

1. Ferguson, B.; Zhang, X.-C. Materials for terahertz science and technology. Nat. Mater. 2002, 1, 26-33. [CrossRef]

2. Lee, Y. Principles of Terahertz Science and Technology; Springer: Berlin, Germany, 2009.

3. Ma, W.; Li, C.; Wang, Z.; Li, L.; Wang, S.; Sun, C. Application of Terahertz Time-Domain Spectroscopy in Characterizing Thin Metal Film-Substrate Structures. IEEE Trans. Terahertz Sci. Technol. 2020, 10, 593-598. [CrossRef]

4. Jepsen, P.; Cooke, D.; Koch, M. Terahertz spectroscopy and imaging-Modern techniques and applications. Laser Photonics Rev. 2010, 5, 124-166. [CrossRef] 
5. Wei, X.; Zhu, S.; Zhou, S.; Zheng, W.; Li, S. Identification of Soybean Origin by Terahertz Spectroscopy and Chemometrics. IEEE Access 2020, 8, 184988-184996. [CrossRef]

6. Federici, J.F.; Schulkin, B.; Huang, F.; Gary, D.; Barat, R.; Oliveira, F.; Zimdars, D. THz imaging and sensing for security applications-explosives, weapons and drugs. Semicond. Sci. Technol. 2005, 20, S226-S280. [CrossRef]

7. Zhang, L.; Zhong, H.; Deng, C.; Zhang, C.; Zhao, Y. Terahertz wave reference-free phase imaging for identification of explosives. Appl. Phys. Lett. 2008, 92, 091117. [CrossRef]

8. Li, R.; Li, C.; Li, H.; Wu, S.; Fang, G. Study of Automatic Detection of Concealed Targets in Passive Terahirtz Images for Intelligent Security Screening. IEEE Trans. Terahertz Sci. Technol. 2019, 9, 165-176. [CrossRef]

9. Zhang, X.; Chang, T.; Wang, Z.; Cui, H.-L. Three-Dimensional Terahertz Continuous Wave Imaging Radar for Nondestructive Testing. IEEE Access 2020, 8, 144259-144276. [CrossRef]

10. Grachev, Y.V.; Liu, X.; Putilin, S.E.; Tsypkin, A.N.; Bespalov, V.G.; Kozlov, S.A.; Zhang, X.-C. Wireless Data Transmission Method Using Pulsed THz Sliced Spectral Supercontinuum. IEEE Photon. Technol. Lett. 2017, 30, 103-106. [CrossRef]

11. Auston, D.H.; Cheung, K.P.; Smith, P.R. Picosecond photoconducting Hertzian dipoles. Appl. Phys. Lett. 1984, 45, 284-286. [CrossRef]

12. Peytavit, E.; Lepilliet, S.; Hindle, F.; Coinon, C.; Akalin, T.; Ducournau, G.; Mouret, G.; Lampin, J.-F. Milliwatt-level output power in the sub-terahertz range generated by photomixing in a GaAs photoconductor. Appl. Phys. Lett. 2011, 99, 223508. [CrossRef]

13. Lepeshov, S.; Gorodetsky, A.; Krasnok, A.; Rafailov, E.; Belov, P. Enhancement of terahertz photoconductive antenna operation by optical nanoantennas. Laser Photonics Rev. 2017, 11, 1600199. [CrossRef]

14. Butcher, P.N.; Cotter, D. The Elements of Nonlinear Optics; Cambridge University Press: Cambridge, UK, 1990.

15. Bashirpour, M.; Forouzmehr, M.; Hosseininejad, S.E.; Kolahdouz, M.; Neshat, M. Improvement of Terahertz Photoconductive Antenna using Optical Antenna Array of ZnO Nanorods. Sci. Rep. 2019, 9, 1-8. [CrossRef]

16. Burford, N.M.; Evans, M.J.; El-Shenawee, M.O. Plasmonic Nanodisk Thin-Film Terahertz Photoconductive Antenna. IEEE Trans. Terahertz Sci. Technol. 2017, 8, 237-247. [CrossRef]

17. Yardimci, N.T.; Yang, S.-H.; Berry, C.W.; Jarrahi, M. High-Power Terahertz Generation Using Large-Area Plasmonic Photoconductive Emitters. IEEE Trans. Terahertz Sci. Technol. 2015, 5, 223-229. [CrossRef]

18. Pouya, T.; Sara, D.; Javad, M. Design and Simulation of a Piezotronic GaN-Based Pulsed THz Emitter. J. Lightwave Technol. 2018, 36, 3645-3651.

19. Ruben, D.; Ríos, R.D.V.; Bikorimana, S.; Dorsinville, R.; Seo, S.W. Terahertz-wave Characterization of LTG-GaAs Thin-film Photoconductive Antenna. IEEE J. Sel. Top. Quantum Electron. 2017, 23, 8500708.

20. Lepeshov, S.; Gorodetsky, A.; Krasnok, A.; Toropov, N.; Vartanyan, T.A.; Belov, P.; Alu, A.; Rafailov, E.U. Boosting terahertz photoconductive antenna performance with optimized plasmonic nanostructures. Sci. Rep. 2018, 8, 6624. [CrossRef]

21. Wang, K.; Gu, J.; Shi, W.; An, Y.; Li, Y.; Tian, Z.; Ouyang, C.; Han, J.; Zhang, W.; Zhen, T. All-dielectric nanograting for increasing terahertz radiation power of photoconductive antennas. Opt. Express 2020, 28, 19144. [CrossRef]

22. Rana, G.; Bhattacharya, A.; Gupta, A.; Ghindani, D.; Jain, R.; Duttagupta, S.P.; Prabhu, S.S. A Polarization-Resolved Study of Nanopatterned Photoconductive Antenna for Enhanced Terahertz Emission. IEEE Trans. Terahertz Sci. Technol. 2019, 9, $193-199$. [CrossRef]

23. Yachmenev, A.E.; Lavrukhin, D.V.; Glinskiy, I.A.; Zenchenko, N.V.; Goncharov, Y.G.; Spektor, I.E.; Khabibullin, R.A.; Otsuji, T.; Ponomarev, D.S. Metallic and dielectric metasurfaces in photoconductive terahertz devices: A review. Opt. Eng. 2019, 59, 061608. [CrossRef]

24. Park, S.-G.; Jin, K.H.; Yi, M.; Ye, J.C.; Ahn, J.; Jeong, K.-H. Enhancement of Terahertz Pulse Emission by Optical Nanoantenna. ACS Nano 2012, 6, 2026-2031. [CrossRef] [PubMed]

25. Liu, S.; Shou, X.; Nahata, A. Coherent Detection of Multiband Terahertz Radiation Using a Surface Plasmon-Polariton Based Photoconductive Antenna. IEEE Trans. Terahertz Sci. Technol. 2011, 1, 412-415. [CrossRef]

26. Heshmat, B.; Pahlevaninezhad, H.; Yuanjie, P.; Masnadi-Shirazi, M.; Lewis, R.B.; Tiedje, T.; Gordon, R.; Darcie, T.E. Nanoplasmonic Terahertz Photoconductive Switch on GaAs. Nano Lett. 2012, 12, 6255-6259. [CrossRef]

27. Berry, C.W.; Hashemi, M.R.; Unlu, M.; Jarrahi, M. Significant Radiation Enhancement in Photoconductive Terahertz Emitters by Incorporating Plasmonic Contact Electrodes. Nat. Commun. 2013, 4, 1622. [CrossRef] [PubMed]

28. Berry, C.W.; Hashemi, M.R.; Jarrahi, M. Generation of high power pulsed terahertz radiation using a plasmonic photo-conductive emitter array with logarithmic spiral antenna. Appl. Phys. Lett. 2014, 104, 081122. [CrossRef]

29. Nguyen, T.K.; Ho, T.A.; Han, H.; Park, I. Numerical Study of Self-Complementary Antenna Characteristics on Substrate Lenses at Terahertz Frequency. J. Infrared Millim. Terahertz Waves 2012, 33, 1123-1137. [CrossRef]

30. Bilal, R.; Rahim, A.A.; Maab, H.; Ali, M.M. Modified Wang Shaped Ultra-Wideband (UWB) Fractal Patch Antenna for MillimetreWave Applications. In Proceedings of the 2018 Progress in Electromagnetics Research Symposium (PIERS-Toyama), Toyama, Japan, 1-4 August 2018.

31. Bilal, R.; Saeed, M.A.; Chaudhary, P.K.; Baqir, M.A.; Kamal, W.; Ali, M.M.; Rahim, A.A. Elliptical metallic rings-shaped fractal metamaterial absorber in the visible regime. Sci. Rep. 2020, 10, 14035. [CrossRef] [PubMed]

32. Nathan, B.M.; Magda, E.O. Review of terahertz photoconductive antenna technology. Opt. Eng. 2017, 56, 010901.

33. Loata, G.C.; Thomson, M.D.; Löffler, T.; Roskos, H.G. Radiation field screening in photoconductive antennae studied via pulsed terahertz emission spectroscopy. Appl. Phys. Lett. 2007, 91, 232506. [CrossRef] 